\title{
Robotic left hepatectomy and Roux-en-Y right hepatico-jejunostomy for biliary papillomatosis
}

\author{
Carmen CW Chu, MB, ChB, FRCSEd, Eric CH Lai *, MB, ChB, FRACS, Oliver CY Chan, MB, ChB, FRCSEd, \\ Daniel TM Chung, MB, ChB, FRCSEd, CN Tang, MB, BS, FRCSEd
}

Department of Surgery, Pamela Youde Nethersole Eastern Hospital, Chai Wan, Hong Kong

*Corresponding author: ericlai@alumni.cuhk.edu.hk

Hong Kong Med J 2016;22:78-80

DOI: 10.12809/hkmj144324

\section{Case report}

An 83-year-old male was referred to us for deranged liver function in December 2010. There was mildly elevated bilirubin level of $36 \mu \mathrm{mol} / \mathrm{L}$, alkaline phosphatase level of $281 \mu \mathrm{mol} / \mathrm{L}$, and transaminase level of $98 \mu \mathrm{mol} / \mathrm{L}$. Tumour markers (carcinoembryonic antigen, alpha-fetoprotein, and carbohydrate antigen 19-9) were normal. Ultrasonography revealed a markedly dilated common bile duct (CBD) and intrahepatic ducts with irregular mural lesions.

Endoscopic retrograde cholangiopancreatography (ERCP) showed a grossly dilated CBD and intrahepatic ducts filled with thick mucus and multiple large filling defects. Brush cytology revealed atypical cells. A nasobiliary drainage catheter was inserted for biliary decompression. Bilatera percutaneous transhepatic biliary drainage (PTBD) catheters were later inserted due to inefficient nasobiliary drainage.

Further evaluation by computed tomographic scan showed dilatation of the whole biliary system with multiple papilloma-like lesions as shown in Figure 1. The presence of mucus in the biliary tree and image findings raised the suspicion of biliary papillomatosis. In order to localise and assess the extent of involvement, intra-operative choledochoscopy was performed via the PTBD tract. The PTBD tract was serially dilated up to 14 Fr to allow passage of a choledochoscope. Multiple biliary papillomas over the left hepatic duct, hilar bifurcation, and upper main bile duct were visualised. The right biliary system, lower CBD, and ampullary region were disease-free. After thorough assessment by intra-operative choledochoscopy, left hepatectomy and main bile duct excision with right hepatico-jejunostomy via robot-assisted laparoscopic approach was performed.

The patient was placed in the reverse Trendelenburg position with legs spread apart, and a 5 - to 12 -mm subumbilical port was inserted with the establishment of carbon dioxide pneumoperitoneum. After diagnostic laparoscopy, five trocars were inserted under direct vision. The extent of disease was assessed by intra-operative ultrasonography (BK Medical, Denmark). The da Vinci S Surgical System (Intuitive Surgical Inc, Sunnyvale [CA], US) was brought into position over the patient's head and docked in. The assistant surgeon stayed on the right side of the patient and performed suction, stapling, and clipping through an assistant port over the right lower quadrant.

The intended extent of parenchymal resection was first marked on the liver surface with electrocautery. The main extrahepatic bile duct was dissected and slung with vascular tape. The right hepatic duct and lower CBD were transected and confirmed to have clear resection margins on frozen section. Porta dissection was performed. The left hepatic artery and left porta vein were dissected and transected. The main and right hepatic artery, and main and right portal vein were identified and protected. Left hemihepatectomy was performed with an ultrasonic surgical aspirator (SonoSurg, SS; Olympus Medical Systems Corporation, Tokyo, Japan) and coagulative scissors, under hemivascular inflow control. Large branches of vascular structures were controlled with endostaplers. A right hepaticojejunostomy via Roux-en-Y reconstruction was fashioned with 3-0 poliglecaprone 25 (Monocryl, Ethicon; Johnson \& Johnson, Amersfoort, The Netherlands) sutures intracorporeally. The sideto-side jejunojejunostomy was performed and the enterotomy site closed intracorporeally with an endostapler and 3-0 monocryl sutures. The specimen (Fig 2) was delivered via the extension of the left abdominal port. Operating time was 367 minutes and operative blood loss was $200 \mathrm{~mL}$. The postoperative course was uneventful and the patient was discharged on postoperative day 13. Histopathological examination revealed extensive papillomatosis with villous adenomatous changes over both left hepatic ducts and the CBD associated with moderate-tosevere dysplasia. There was no evidence of invasion to suggest malignant transformation. At 50-month follow-up, the patient remained disease-free with no further biliary obstruction. 


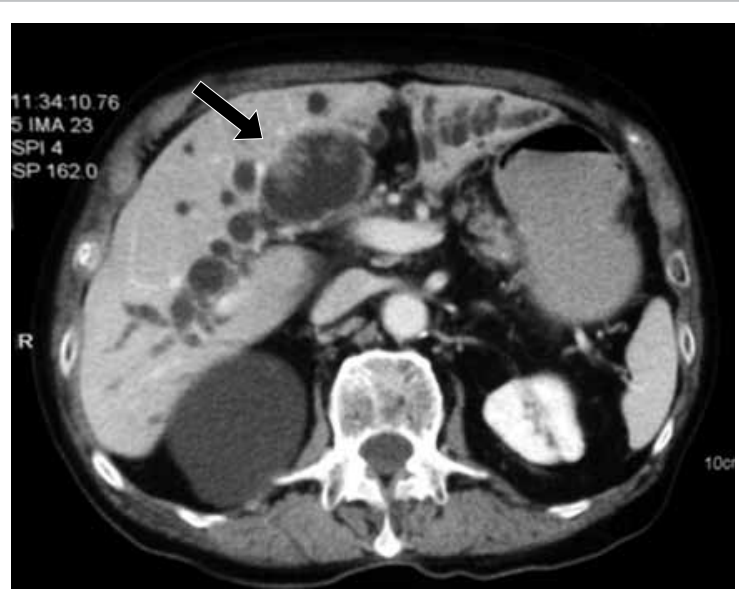

FIG I. Computed tomography showing cystic dilatation of biliary tree with intramural components (arrow)

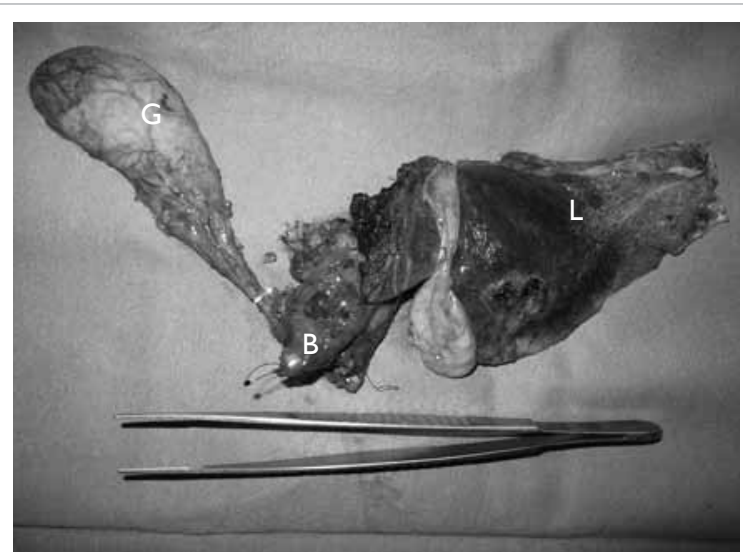

FIG 2. Resected specimen comprising gall bladder (G), left hemi liver ( $L$ ), and common bile duct (B)

\section{Discussion}

Biliary papillomatosis is a rare condition characterised by papillary proliferation of the lining columnar epithelium of the bile ducts. It was first reported by Chappet $^{1}$ in 1894 and was initially thought to be an entity with low malignant potential. Malignant transformation, however, is noted in $20 \%$ to $50 \%$ of cases as a consequence of adenoma carcinoma sequence. ${ }^{2,3}$

Middle-aged to elderly patients are commonly affected with a slight male predominance. The clinical presentation varies from being asymptomatic, as in our patient, to the presence of recurrent abdominal pain, obstructive jaundice, or recurrent cholangitis due to biliary obstruction by mucus.

The exact pathogenesis remains to be elucidated, but biliary papillomatosis is associated with conditions such as recurrent pyogenic cholangitis and congenital choledochal cyst. It has been postulated that longstanding irritation by stones or infection stimulates reactive hyperplasia with subsequent dysplasia in the biliary system.

Despite considerable improvements in imaging techniques, diagnosis remains a challenge as the presenting symptoms are more commonly caused by choledocholithiasis. Ultrasonography and computed tomography reveal intrabiliary masses with cystic dilatation in the proximal biliary tree. On the other hand, ERCP or magnetic resonance cholangiopancreatography shows an irregular filling defect that causes obstruction with proximal dilatation. The presence of mucobilia should raise the suspicion of biliary papillomatosis.

Histopathological examination reveals that the biliary system is often replaced by velvety papillary growth that possesses a fibrovascular core lined by columnar epithelium with varying degrees of cellular atypia.

Management is difficult due to the diffuse nature of the disease. A range of treatment strategies that include local ablation, photodynamic therapy, radical excision, or total hepatectomy with liver transplantation have been reported. Local ablative therapy and curettage, and radical excision with clear resection margins are associated with better survival. ${ }^{2,4}$

Accurate localisation and assessment of the extent of disease involvement remains pertinent to an accurate choice of treatment. In a Korean series by Lee et al, ${ }^{2}$ curative resection was associated with significantly better survival (60 months in curative resection vs 36 months in palliative surgery group); similar results were demonstrated by another series of 18 cases in China. ${ }^{5}$ Due to the high propensity for diffuse involvement, recurrence and malignant transformation, timely diagnosis and radical resection remain the cornerstone for successful treatment.

The advent of robotic surgery has brought about revolutionary changes in current surgical developments, but the feasibility and benefits in hepatobiliary surgeries are yet to be explored. Deep anatomical locations, complex vascularity, and large organ volume in hepatobiliary surgery often pose a challenge to the conventional laparoscopic approach. Such hurdles can be overcome by robotic surgery: recent studies have shown that a robotassisted approach offers a safe and feasible option for hepatobiliary surgery with promising results. ${ }^{6-10}$ The enhanced dexterity of EndoWrist (Intuitive Surgical Inc, Sunnyvale [CA], US), with its 7 degrees of freedom of movement, allows meticulous dissection and precise tissue handling, enabling intracorporeal suturing even in the most technically demanding areas that would otherwise be impossible to access in conventional laparoscopic surgery. The three-dimensional stereoscopic video system with 
magnification enhances visualisation and depth perception. The third robotic arm also allows better organ retraction. The improving technical abilities of the robotic system for dissection and suturing extend the indications of minimally invasive liver surgery to liver resection requiring a biliary reconstruction. Without any doubt, the operation described in this case requires high technical skill and experience, and cannot be quickly introduced into routine practice. We performed this operation following accumulation of 10 years' experience of conventional laparoscopic liver surgery and 2 years' experience of robotic liver resection and robotic biliary surgery. ${ }^{8-12}$ Nonetheless, we believe that with the increasing popularity of robotic surgery, the required skill can be acquired with time. With the help of a robotic system, unilateral hepatico-jejunostomy reconstruction and porta structure dissection can be performed more easily than with the conventional laparoscopic technique.

In conclusion, the robotic approach to treatment of biliary papillomatosis is feasible and safe in selected patients. It also has the advantage of being minimally invasive.

\section{References}

1. Chappet V. Cancer epithelial primitif du canal cholédoque. Lyon Med 1894;76:145-57.

2. Lee SS, Kim MH, Lee SK, et al. Clinicopathologic review of 58 patients with biliary papillomatosis. Cancer 2004;100:783-93.

3. Wu SD, Lu CD, Lu CJ, Huang J, Zhou J. Mucinproducing intrahepatic biliary papillomatosis. Surg Today 2010;40:845-50.

4. Ludwig L, Büchler P, Kleeff J, et al. Multidisciplinary treatment of aggressive and rapidly progressing biliary papillomatosis. Dig Dis Sci 2010;55:3627-9.

5. Jiang L, Yan LN, Jiang LS, et al. Biliary papillomatosis: analysis of 18 cases. Chin Med J (Engl) 2008;121:2610-2.

6. Giulianotti PC, Sbrana F, Coratti A, et al. Totally robotic right hepatectomy: surgical technique and outcomes. Arch Surg 2011;146:844-50.

7. Choi GH, Choi SH, Kim SH, et al. Robotic liver resection: technique and results of 30 consecutive procedures. Surg Endosc 2012;26:2247-58.

8. Lai EC, Tang CN, Yang GP, Li MK. Multimodality laparoscopic liver resection for hepatic malignancy-from conventional total laparoscopic approach to robot-assisted laparoscopic approach. Int J Surg 2011;9:324-8.

9. Lai EC, Tang CN, Li MK. Robot-assisted laparoscopic hemi-hepatectomy: technique and surgical outcomes. Int J Surg 2012;10:11-5.

10. Lai EC, Yang GP, Tang CN. Robot-assisted laparoscopic liver resection for hepatocellular carcinoma: short-term outcome. Am J Surg 2013;205:697-702.

11. Lai EC, Tang CN, Ha JP, Li MK. Laparoscopic liver resection for hepatocellular carcinoma: ten-year experience in a single center. Arch Surg 2009;144:143-7; discussion 148.

12. Lai EC, Tang CN, Yang GP, Li MK. Minimally invasive surgical treatment of hepatocellular carcinoma: long-term outcome. World J Surg 2009;33:2150-4. 